\title{
Perfil da Intervenção Coronária Percutânea no Infarto Agudo do Miocárdio com Supradesnivelamento do Segmento ST no Brasil de 2006 a 2010 - Registro CENIC
}

\author{
Bruno da Silva Matte', Luiz Carlos Corsetti Bergoli', Julise Arpini Balvedi', \\ Alexandre do Canto Zago*1, em nome dos colaboradores do Registro CENIC
}

\begin{abstract}
RESUMO
Introdução: $\mathrm{O}$ infarto agudo do miocárdio (IAM) persiste como importante causa de morbidade e mortalidade. Este estudo visa a delinear o panorama nacional da intervenção coronária percutânea (ICP) no cenário do IAM, analisando diferentes períodos e regiões do Brasil, com enfoque na ICP primária e nos tratamentos adjuntos farmacológicos e mecânicos. Métodos: Foram analisados dados de 20.004 pacientes com diagnóstico de IAM com supradesnivelamento do segmento ST (IAMCSST) e submetidos a ICP, provenientes do Registro CENIC (Central Nacional de Intervenções Cardiovasculares), de janeiro de 2006 a dezembro de 2010. Esses dados são oriundos de 252 centros localizados em 22 Estados das cinco regiões do País. Resultados: A ICP primária correspondeu a $57,8 \%$ das ICPs realizadas no contexto do IAM, seguida de ICP eletiva pós-IAMCSST $(35,7 \%)$, ICP de resgate $(6,1 \%)$ e ICP facilitada $(0,4 \%)$. A evolução ao longo dos anos evidencia aumento progressivo do número de ICPs primárias no Brasil, partindo de $56,7 \%$ do total em 2006 para $71,6 \%$ em 2010. O tempo médio porta-balão da ICP primária no Brasil nesse período foi de 2 horas. A aspiração de trombos aumentou de 0,4\% em 2006 para 8,2\% dos casos em 2010. A taxa média de sucesso do procedimento foi de $93,8 \%$, enquanto a de óbito hospitalar foi de apenas 2,8\%. Conclusões: A ICP no cenário do IAMCSST vem apresentando avanços de 2006 a 2010, embora de maneira heterogênea nas diferentes regiões do Brasil, mediante aumento das taxas de ICP primária e maior utilização de dispositivos de aspiração de trombo, os quais ainda não foram incorporados na rotina. Investimentos em recursos humanos e implementação de protocolos de atendimento constituem elementos essenciais para a otimi-
\end{abstract}

\section{ABSTRACT}

National Profile of Percutaneous Coronary Intervention in Acute ST Elevation Myocardial Infarction in Brazil from 2006 to 2010 the CENIC Registry

Background: Acute myocardial infarction (AMI) remains a major cause of morbidity and mortality. This study aims to outline the national profile of percutaneous coronary intervention $(\mathrm{PCl})$ in the setting of $\mathrm{AMI}$, analyzing different time periods and geographic regions, with focus on primary $\mathrm{PCl}$ and adjunctive pharmacological and mechanical treatments. Methods: Data from 20,004 patients with ST elevation myocardial infarction (STEMI) undergoing $\mathrm{PCl}$ and included in the CENIC Registry (National Center of Cardiovascular Interventions) from January 2006 to December 2010 were included in this study. Data were obtained from 252 centers located in 22 states from five different geographic regions in the country. Results: Primary $\mathrm{PCl}$ accounted for $57.8 \%$ of $\mathrm{PCl}$ performed in the setting of $\mathrm{AMI}$, followed by elective $\mathrm{PCl}$ after STEMI $(35.7 \%)$, rescue $\mathrm{PCl}(6.1 \%)$ and facilitated $\mathrm{PCl}(0.4 \%)$. The evolution over time showed a progressive increase in the number of primary PCls in Brazil, from $56.7 \%$ in 2006 to $71.6 \%$ in 2010. The mean door-to-balloon time of primary $\mathrm{PCl}$ in Brazil during this period was 2 hours. Thrombus aspiration increased from $0.4 \%$ in 2006 to $8.2 \%$ of cases in 2010. Procedural success rate was $93.8 \%$, while in-hospital mortality was only $2.8 \%$. Conclusions: $\mathrm{PCl}$ in the setting of STEMI has improved from 2006 to 2010, although heterogeneously in the different regions of Brazil, due to increased primary $\mathrm{PCl}$ rates and higher use of thrombus aspiration devices, which have not been incorporated in

\footnotetext{
* Coordenador do Registro CENIC (Central Nacional de Intervenções Cardiovasculares).

1 Hospital de Clínicas de Porto Alegre - Porto Alegre, RS, Brasil. Correspondência: Alexandre do Canto Zago. Rua Ramiro Barcelos, 2.350 - 2o andar - Porto Alegre, RS, Brasil - CEP 90035-003 E-mail: zagoac@uol.com.br

Recebido em: 11/4/2011 • Aceito em: 3/6/2011
} 
zação do tempo porta-balão e para a melhora dos resultados clínicos.

DESCRITORES: Infarto do miocárdio. Angioplastia. Stents. Sistema de registros. Protocolos clínicos.

0 infarto agudo do miocárdio (IAM) persiste como importante causa de morbidade e mortalidade, apesar dos avanços tanto no tratamento como na prevenção dessa condição, acarretando grandes custos aos sistemas de saúde. ${ }^{1}$ O tratamento do IAM com supradesnivelamento do segmento ST (IAMCSST) tem apresentado melhores resultados desde o advento da intervenção coronária percutânea (ICP) primária, hoje método de reperfusão de escolha, que promoveu redução significativa das taxas de mortalidade, reinfarto e acidente vascular cerebral em comparação ao tratamento com fibrinolíticos..$^{2-8}$ Esse benefício está relacionado a vários fatores, como maior taxa de reperfusão mecânica (cerca de 90\%) quando comparada à da reperfusão farmacológica com fibrinolíticos (cerca de 50\%), possibilidade de simultaneamente tratar a estenose subjacente, e menor taxa de complicações hemorrágicas associadas. ${ }^{9}$

A ICP no contexto do IAMCSST pode ser dividida em primária (sem uso prévio de fibrinolíticos), facilitada (relacionada à utilização de farmacologia prévia), de salvamento ou resgate (após insucesso de fibrinólise química), e aquela praticada de maneira eletiva após a fibrinólise. ${ }^{10}$

Os dados apresentados neste estudo visam a delinear o panorama nacional da ICP no cenário do IAM, analisando diferentes períodos e regiões do Brasil. Enfoque é dado à ICP primária e aos tratamentos adjuntos farmacológicos ou mecânicos, como o uso de dispositivos de aspiração de trombos e de fármacos inibidores da glicoproteína Ilb/IIla, comparando a prática observada com as diretrizes atuais da Sociedade Brasileira de Hemodinâmica e Cardiologia Intervencionista $(\mathrm{SBHCl})$ e de outras sociedades internacionais.

\section{MÉTODOS}

Os dados utilizados neste estudo foram obtidos do Registro CENIC (Central Nacional de Intervenções Cardiovasculares), instituído pela SBHCl em 1993, com o objetivo principal de coletar os dados acerca dos procedimentos intervencionistas realizados no País. Esses dados foram coletados por cardiologistas intervencionistas vinculados à $\mathrm{SBHCl}$ e registrados em ficha de coleta de dados padronizada, com entrada para variáveis relacionadas a perfil clínico, uso prévio de trombolítico, tempo porta-balão, características angiográficas e relativas ao procedimento, e resultados clínicos hospitalares. Essas fichas são enviadas de forma voluntária the routine practice. Investments in staff training and implementation of clinical protocols are essential to optimize the door-to-balloon time and improve clinical outcomes.

KEY-WORDS: Myocardial infarction. Angioplasty. Stents. Registries. Clinical protocols.

ao centro coordenador da CENIC, localizado na sede da $\mathrm{SBHCl}$, em São Paulo (SP), de forma que os registros enviados não correspondem à totalidade de procedimentos realizados em todo o País.

De janeiro de 2006 a dezembro de 2010, foram enviados dados de 20.004 pacientes com diagnóstico de IAMCSST e submetidos a ICP. Esses dados são provenientes de 252 centros localizados em 22 Estados das cinco regiões do Brasil. O diagnóstico e o tratamento do IAMCSST em cada centro colaborador foram feitos de acordo com as rotinas específicas, a critério das equipes clínicas e do cardiologista intervencionista. Todas as fichas enviadas nesse período que indicavam a realização de ICP em pacientes com diagnóstico de IAMCSST foram analisadas. Os resultados estão apresentados na forma de tabelas, com valores porcentuais expressando a média nacional e estratificados por região geográfica e/ou período.

\section{RESULTADOS}

Do total de pacientes cujos dados sobre IAM foram enviados ao Registro CENIC, 13.762 (68,8\%) pacientes tiveram seus procedimentos realizados na região Sudeste do Brasil, enquanto a região Norte contribuiu com apenas 134 casos $(0,7 \%)$. Em relação ao perfil dos pacientes no Brasil, houve predomínio do sexo masculino $(69,9 \%)$, a média de idade foi de 60,8 anos e a hipertensão arterial sistêmica foi o fator de risco mais prevalente, seguida de dislipidemia, tabagismo, história familiar positiva e diabetes melito. No momento da admissão hospitalar, a apresentação dos pacientes em classe Killip I foi a mais prevalente, constituindo 76,5\% dos casos, enquanto apenas $4,8 \%$ dos pacientes apresentaram choque cardiogênico (Killip IV) (Tabela 1).

Na Tabela 2 constam os dados sobre as características da ICP. No Brasil, a ICP primária correspondeu a $57,8 \%$ das ICPs realizadas no contexto do IAM, enquanto a ICP eletiva pós-IAMCSST foi realizada em 35,7\% dos casos. A ICP de resgate e a ICP facilitada constituíram $6,1 \%$ e $0,4 \%$ do total de casos relatados, respectivamente. A região Sul é a que mais realizou ICPs primárias $(79,4 \%)$, seguida das regiões Nordeste, Sudeste, Centro-Oeste e Norte. O tempo médio porta-balão da ICP primária no Brasil foi de 2 horas, variando de 1,7 hora no Nordeste a 2,3 horas no Sudeste. Nos casos em que foi realizada angioplastia eletiva pós-IAMCSST, o tempo médio foi de 8,4 dias. 
TABELA 1

Perfil dos pacientes submetidos a intervenção coronária percutânea no infarto agudo do miocárdio com supradesnivelamento do segmento ST, nas diferentes regiões do Brasil

\begin{tabular}{|c|c|c|c|c|c|c|}
\hline & $\begin{array}{l}\text { Centro-Oeste } \\
(n=1.460)\end{array}$ & $\begin{array}{c}\text { Nordeste } \\
(n=2.409)\end{array}$ & $\begin{array}{c}\text { Norte } \\
(n=134)\end{array}$ & $\begin{array}{c}\text { Sudeste } \\
(\mathrm{n}=13.762)\end{array}$ & $\begin{array}{c}\text { Sul } \\
(\mathrm{n}=2.239)\end{array}$ & $\begin{array}{c}\text { Brasil } \\
(n=20.004)\end{array}$ \\
\hline Número de pacientes, \% & 7,3 & 12 & 0,7 & 68,8 & 11,2 & 100 \\
\hline Sexo masculino, $\%$ & 73 & 65,9 & 69,4 & 70,5 & 68,6 & 69,9 \\
\hline Média de idade, anos & 60,1 & 61,8 & 60,8 & 60,2 & 60,8 & 60,8 \\
\hline História familiar, \% & 26 & 31 & 20,1 & 23,1 & 19,3 & 23,8 \\
\hline Hipertensão, \% & 65,8 & 76 & 78,4 & 74,6 & 60 & 72,5 \\
\hline Tabagismo, \% & 35,3 & 33,4 & 34,3 & 39,5 & 35,7 & 38 \\
\hline Dislipidemia, \% & 46,6 & 54,8 & 53,7 & 47,9 & 51 & 49 \\
\hline Diabetes melito, $\%$ & 20,7 & 22,2 & 18,7 & 20,4 & 15,4 & 20,1 \\
\hline CRM prévia, \% & 1,8 & 2,6 & 1,5 & 2,6 & 2,7 & 2,6 \\
\hline ICP prévia, \% & 7,4 & 9,3 & 6 & 8,1 & 12,5 & 8,7 \\
\hline IAM prévio, \% & 13,6 & 10 & 31,3 & 14,1 & 12,5 & 13,5 \\
\hline \multicolumn{7}{|l|}{ Classe de Killip, \% } \\
\hline I & 80,9 & 81,5 & 80,6 & 74,1 & 83,1 & 76,5 \\
\hline II & 9,7 & 9,8 & 11,9 & 17 & 7,2 & 14,5 \\
\hline III & 4,9 & 3,2 & 3,7 & 4,5 & 3,1 & 4,2 \\
\hline IV & 4,6 & 5,5 & 3,7 & 4,4 & 6,6 & 4,8 \\
\hline
\end{tabular}

CRM = cirurgia de revascularização miocárdica; $I \mathrm{AM}$ = infarto agudo do miocárdio; $\mathrm{ICP}$ = intervenção coronária percutânea; $\mathrm{n}=$ número de pacientes.

TABELA 2

Características da intervenção coronária percutânea realizada

\begin{tabular}{|c|c|c|c|c|c|c|}
\hline & Centro-Oeste & Nordeste & Norte & Sudeste & Sul & Brasil \\
\hline \multicolumn{7}{|l|}{$\mathrm{ICP}, \%$} \\
\hline Primária & 49,7 & 77 & 48,4 & 51,7 & 79,4 & 57,8 \\
\hline Eletiva & 46,4 & 19,7 & 51,6 & 40,3 & 17,3 & 35,7 \\
\hline Facilitada & 0,1 & 0,3 & 0 & 0,4 & 0,5 & 0,4 \\
\hline Resgate & 3,8 & 3 & 0 & 7,6 & 2,7 & 6,1 \\
\hline Uso de trombolítico prévio, \% & 20,7 & 5,6 & 19 & 18,7 & 5,1 & 15,7 \\
\hline \multicolumn{7}{|l|}{ Tempo médio para ICP } \\
\hline Tempo porta-balão na ICP primária, horas & 2,2 & 1,7 & 2,1 & 2,3 & 1,8 & 2 \\
\hline Angioplastia eletiva, dias & 8,8 & 8,3 & 10,3 & 7,1 & 7,5 & 8,4 \\
\hline
\end{tabular}

A evolução ao longo dos anos evidencia aumento progressivo do número de ICPs primárias no Brasil, partindo de $56,7 \%$ do total de ICPs realizadas no contexto de IAM em 2006 para 71,6\% do total de ICPs em pacientes com IAMCSST em 2010, atingindo o porcentual de 93,6\% na região Sul em 2010 (Tabela 3).

Em relação à artéria culpada, houve distribuição homogênea entre as regiões. A artéria descendente anterior esquerda foi o vaso mais frequentemente responsável por IAMCSST (43,3\%), seguida da artéria coro- nária direita $(35,1 \%)$ e da artéria circunflexa (10\%) (Tabela 4). Quanto ao tipo de stent utilizado, evidenciou-se o uso de stents convencionais em 96,3\% e de stents farmacológicos em 3,7\% das ICPs no contexto do IAM no Brasil. Em relação às diferentes regiões, observou-se que o porcentual de implante em pacientes com IAMCSST variou de 3,1\% na região Norte a 8,9\% na região Centro-Oeste (Tabela 5).

Em 2006, a aspiração de trombos foi realizada em 0,4\% dos casos, passando para 3,4\% em 2008, 7,2\% em 
2009 e atingindo 8,2\% em 2010, sendo as regiões Sul e Sudeste as principais responsáveis pelo incremento do uso desse dispositivo (Tabela 6). Em relação ao uso de outros dispositivos, observou-se a utilização de filtros de proteção distal em pequeno número de pacientes submetidos a ICP no cenário do IAM (0,1\%). Ultrassom intracoronário foi empregado durante o procedimento em $0,3 \%$ dos casos (Tabela 7 ).

Inibidores da glicoproteína Ilb/IIla foram administrados a $15,8 \%$ do total de ICPs realizadas no contexto do IAM, sendo tirofiban utilizado em $12,3 \%$ dos casos e abciximab, em apenas 3,5\% dos casos (Tabela 8).

A taxa média de sucesso clínico de ICP em pacientes com IAMCSST no Brasil foi de 93,8\%, sendo essa taxa bastante homogênea entre as cinco regiões do País. A taxa média de óbito hospitalar foi de apenas 2,8\% (Tabela 9).

\section{DISCUSSÃO}

Os dados apresentados constituem uma consistente e atualizada fonte de dados sobre a realidade nacional do tratamento percutâneo do IAMCSST, visto que foram analisados dados do Registro CENIC de todas as regiões do País coletados ao longo dos últimos cinco anos. Os resultados evidenciam a necessidade de melhorias na qualidade assistencial, em decorrência, principalmente, das taxas ainda baixas de ICPs primárias, principalmente nas regiões Norte, Centro-Oes-

TABELA 3

Intervenção coronária percutânea primária: evolução porcentual por região - 2006 a 2010

\begin{tabular}{ccccccc}
\hline $\begin{array}{c}\text { Centro-Oeste } \\
(\boldsymbol{\%})\end{array}$ & $\begin{array}{c}\text { Nordeste } \\
(\boldsymbol{\%})\end{array}$ & $\begin{array}{c}\text { Norte } \\
(\boldsymbol{\%})\end{array}$ & $\begin{array}{c}\text { Sudeste } \\
(\boldsymbol{\%})\end{array}$ & $\begin{array}{c}\text { Sul } \\
(\boldsymbol{\%})\end{array}$ & $\begin{array}{c}\text { Brasil } \\
(\boldsymbol{\%})\end{array}$ \\
\hline 2006 & 51,4 & 73,5 & 3,7 & 51,4 & 67,3 & 56,7 \\
2007 & 57,2 & 80,9 & 0 & 45,9 & 79,3 & 53,4 \\
2008 & 53,2 & 80,8 & 55,6 & 53,4 & 81,8 & 60,1 \\
2009 & 46,5 & 80,2 & 48,5 & 55,3 & 89,7 & 61,2 \\
2010 & 44,1 & 68,4 & 22,7 & 56,9 & 93,6 & 71,6 \\
\hline
\end{tabular}

TABELA 4

Vaso responsável pelo infarto agudo do miocárdio com supradesnivelamento do segmento ST - 2006 a 2010

\begin{tabular}{lcccccc}
\hline & Centro-Oeste & Nordeste & Norte & Sudeste & Sul & Brasil \\
\hline Tronco de coronária esquerda, \% & 0,9 & 1,1 & 1,3 & 0,6 & 0,6 & 0,7 \\
Descendente anterior esquerda, \% & 43 & 44,1 & 48 & 43,1 & 43,2 & 43,3 \\
Ramo diagonal, \% & 3,1 & 3,6 & 2 & 3,3 & 4,4 & 3,5 \\
Circunflexa, \% & 9,8 & 9,4 & 7,3 & 10,3 & 9,2 & 10 \\
Ramo marginal, \% & 3,6 & 3,2 & 1,3 & 3,6 & 4,1 & 3,5 \\
Coronária direita, \% & 35,1 & 35 & 38,7 & 35,2 & 34 & 35,1 \\
Artéria torácica interna esquerda, \% & 0 & 0 & 0 & 0 & 0 & 0 \\
Ponte de safena, \% & & & & & & \\
$\quad$ Aorta-descendente anterior esquerda & 0 & 0 & 0 & 0 & 0,1 & 0 \\
$\quad$ Aorta-ramo marginal & 0 & 0 & 0 & 0 & 0 & 0 \\
$\quad$ Aorta-coronária direita & 0,1 & 0,1 & 0 & 0,2 & 0 & 0,1 \\
Enxerto radial, \% & 0 & 0 & 0 & 0 & 0 & 0 \\
Outros, \% & 4,4 & 3,5 & 1,4 & 3,7 & 4,4 & 3,8 \\
\hline
\end{tabular}

TABELA 5

Tipos de stent utilizados

\begin{tabular}{lcccccc}
\hline & Centro-Oeste & Nordeste & Norte & Sudeste & Sul & Brasil \\
\hline Convencionais, \% & 91,1 & 92,3 & 96,9 & 95,6 & 96,3 & 96,3 \\
Farmacológicos, \% & 8,9 & 7,7 & 3,1 & 4,4 & 3,7 & 3,7 \\
\hline
\end{tabular}


TABELA 6

Dispositivos de aspiração de trombos utilizados na intervenção coronária percutânea primária tratada com implante de stents: evolução porcentual por região - 2006 a 2010

\begin{tabular}{|c|c|c|c|c|c|c|}
\hline & $\begin{array}{c}\text { Centro-Oeste } \\
(\%)\end{array}$ & $\begin{array}{c}\text { Nordeste } \\
(\%)\end{array}$ & $\begin{array}{c}\text { Norte } \\
(\%)\end{array}$ & $\begin{array}{l}\text { Sudeste } \\
(\%)\end{array}$ & $\begin{array}{l}\text { Sul } \\
(\%)\end{array}$ & $\begin{array}{c}\text { Brasil } \\
(\%)\end{array}$ \\
\hline 2006 & 0,9 & 0 & ND & 0,5 & 0 & 0,4 \\
\hline 2007 & 1,1 & 0,4 & ND & 1,6 & 2,1 & 1,7 \\
\hline 2008 & 0 & 3 & 0 & 4,5 & 1,5 & 3,4 \\
\hline 2009 & 1,3 & 2,2 & 0 & 7,2 & 7,2 & 7,2 \\
\hline 2010 & 0 & 1,2 & 0 & 8,1 & 11,3 & 8,2 \\
\hline
\end{tabular}

TABELA 7

Dispositivos adjuntos utilizados na intervenção coronária percutânea

\begin{tabular}{lcccccc}
\hline & Centro-Oeste & Nordeste & Norte & Sudeste & Sul & Brasil \\
\hline Aterectomia, \% & 0 & 0 & 0 & 2,4 & 0 & 1,6 \\
Braquiterapia, \% & 0 & 0 & 0 & 0 & 0 & 0 \\
Cutting balloon, \% & 0,2 & 0 & 0 & 0,5 & 0,6 & 0,5 \\
Dispositivo de proteção distal, \% & 0 & 0 & 0 & 0,1 & 0,3 & 0,1 \\
Pressure wire, \% & 0 & 0 & 0 & 1 & 0 & 0,7 \\
Rotablator, \% & 0 & 0 & 0 & 0 & 0,1 & 0 \\
Monitoração com ultrassonografia & 0,2 & 0 & 0 & 0,2 & 0,8 & 0,3 \\
intracoronária, \% & & & & & & \\
\hline
\end{tabular}

TABELA 8

Uso de inibidores da glicoproteína IIb/IIla na intervenção coronária percutânea

\begin{tabular}{lcccccc}
\hline & Centro-Oeste & Nordeste & Norte & Sudeste & Sul & Brasil \\
\hline Abciximab, \% & 3,1 & 3,4 & 0 & 3,8 & 2,2 & 3,5 \\
Tirofiban, \% & 11,5 & 7,6 & 0 & 13,4 & 11,7 & 12,3 \\
Total, \% & 14,6 & 11 & 0 & 17,3 & 13,9 & 15,8 \\
\hline
\end{tabular}

TABELA 9

Resultados do procedimento

\begin{tabular}{lcccccc}
\hline & Centro-Oeste & Nordeste & Norte & Sudeste & Sul & Brasil \\
\hline Sucesso clínico, n (\%) & $1.307(93)$ & $2.311(95,9)$ & $128(95,5)$ & $12.923(93,9)$ & $2.103(91,7)$ & $18.772(93,8)$ \\
Reinfarto, \% & 1,7 & 0,2 & 0 & 0,5 & 0,7 & 0,6 \\
Óbito hospitalar, \% & 2,8 & 1,5 & 4,5 & 2,9 & 4,4 & 2,8 \\
Complicações, \% & 0 & 0 & 0 & 0 & 0 & 0 \\
Insuficiência renal aguda & 0,6 & 0,1 & 0,7 & 0,2 & 0,3 & 0,2 \\
Vasculares menores & 0,9 & 0 & 0,7 & 2,9 & 0,4 & 2,1 \\
Vasculares maiores & 0,1 & 0,1 & 0,7 & 2,6 & 0,3 & 1,8 \\
AVC hemorrágico & 0,1 & 0 & 0 & 0 & 0,1 & 0 \\
AVC isquêmico & 0,3 & 0,1 & 0 & 0,1 & 0,1 & 0,1 \\
Total & 1,8 & 0,2 & 1,5 & 3,3 & 1 & 2,5 \\
\hline
\end{tabular}

AVC = acidente vascular cerebral; $\mathrm{n}$ = número de pacientes. 
te e Sudeste. O caráter observacional deste estudo não permite conclusões sobre as causas dessas discrepâncias, que, presumidamente, são multifatoriais e incluem as longas distâncias e o pequeno número de centros com laboratórios de hemodinâmica disponíveis nas regiões Norte e Centro-Oeste. Na região Sudeste, causam surpresa os baixos porcentuais de ICP primária, uma vez que a região possui grande número de hospitais com serviços de cardiologia invasiva.

Ao se analisar isoladamente cada região, observase que a região Centro-Oeste não avançou e até reduziu o porcentual de realização de ICPs primárias, enquanto a região Sudeste apresentou pequeno avanço ao longo dos anos. A região Norte apresentou valores muito baixos em 2006 e 2007, ocorrendo significativo avanço em 2008 e 2009, seguido de retração para aproximadamente $50 \%$ de ICPs primárias em 2010. A região Nordeste manteve porcentual constante e aceitável de aproximadamente $70 \%$ a $80 \%$ ao longo dos últimos cinco anos. Na região Sul, houve avanço significativo e progressivo no período observado, atingindo 93,6\% dos casos em 2010, o que demonstra a viabilidade de amplo acesso a essa modalidade de tratamento padrão para o IAMCSST em nosso meio.

O tempo médio porta-balão observado no Brasil foi de 120 minutos, embora o tempo preconizado para ICP primária pela diretriz da $\mathrm{SBHCl}$ seja de 90 minutos após o diagnóstico de IAM. A região Nordeste é a que mais se aproximou do tempo porta-balão recomendado e a região Sudeste, a que mais se distanciou dessa meta; entretanto, nenhuma região atingiu o tempo ideal. Esse retardo provavelmente decorre da demora no diagnóstico do IAMCSST e da ausência ou da não aplicação adequada de protocolos sistematizados de atendimento à dor torácica. Portanto, treinamento das equipes em atendimento à dor torácica, implementação de protocolos de manejo inicial com rotinas estabelecidas e contato imediato com cardiologistas intervencionistas constituem elementos essenciais para otimização do tempo porta-balão. Deve-se ressaltar que a demora do paciente em procurar atendimento médico e o retardo na transferência de pacientes com diagnóstico de IAMCSST para centros equipados com laboratório de hemodinâmica também são fatores que contribuem significativamente para o retardo do tratamento do IAMCSST, com impacto direto nas taxas de morbidade e mortalidade.

O uso de stents farmacológicos para o tratamento do IAMCSST é ainda tema controverso. A comparação entre stents farmacológicos e não-farmacológicos no cenário da ICP primária foi realizada em 11 estudos controlados, que não demonstraram diferenças significativas no seguimento clínico de até dois anos em relação a mortalidade (4,1\% vs. 4,4\%; P = NS), reinfarto $(3,1 \%$ vs. 3,4\%; P = NS) e trombose intrastent $(1,6 \%$ vs. $2,2 \% ; P=N S)$. Contudo, houve redução das taxas de revascularização da lesãoalvo [5,1\% vs. $12,6 \%$; risco relativo (RR) 0,36 ; $\mathrm{P}<0,001] .{ }^{11}$ A baixa prevalência de uso de stents farmacológicos observada $(<10 \%$ em todo o País) provavelmente justifica-se pela falta de evidências em relação à redução de mortalidade e reinfarto, associada à necessidade de rápida tomada de decisão durante esse procedimento de caráter emergencial, o que limita o tempo para a análise detalhada do histórico clínico do paciente e para a solução de questões de cunho burocrático. ${ }^{12}$

A aspiração de trombos durante ICP primária tem sido preconizada como procedimento padrão no tratamento de pacientes com IAMCSST, resultando em meIhores taxas de reperfusão e de desfechos clínicos em relação à ICP sem aspiração de trombos. A partir de 2008, quando surgiram evidências consistentes para sua recomendação rotineira nesse cenário com a publicação de ensaios clínicos desenhados especificamente para a avaliação desse dispositivo ${ }^{13-15}$, houve aumento significativo em apenas algumas regiões, como Sul e Sudeste. Tendo em vista os benefícios clínicos e angiográficos demonstrados por esses dispositivos, seu uso na prática atual $(8,2 \%)$ ainda é muito abaixo do ideal e deve ser fortemente encorajado.

Em relação aos dispositivos de proteção distal, uma metanálise ${ }^{16}$ que incluiu oito estudos randomizados não mostrou diferença na normalização do fluxo sanguíneo no vaso epicárdico culpado em pacientes alocados para uso de proteção distal em relação ao grupo controle. Portanto, seu uso rotineiro não é encorajado para ICP no contexto do IAMCSST (recomendação classe IIb pela Diretriz de Intervenção Coronária Percutânea da $\mathrm{SBHCl})^{12}$, o que está sendo seguido pelos operadores brasileiros, conforme os dados da CENIC.

Embora não haja consenso em relação ao uso dos inibidores da glicoproteína IIb/IIla no IAM, observou-se porcentual de uso em $15,8 \%$ dos casos, o que pode ser justificado por sua utilização em algumas situações específicas, como presença de grande carga trombótica visível ou inibição plaquetária com tienopiridínicos não efetiva no momento da ICP. ${ }^{17}$ A despeito de o abciximab apresentar maior nível de evidência para uso nesse cenário $^{18}$, observa-se maior prevalência de uso de tirofiban, o que provavelmente se deve a seu menor custo.

Os dados relatados demonstram alta taxa de sucesso clínico $(93,8 \%)$ e baixa taxa de óbito hospitalar $(2,8 \%)$ e de complicações (2,5\%). Entretanto, deve-se observar que este estudo compreende um registro com preenchimento dos dados geralmente no período pós-procedimento imediato, o que pode subestimar as taxas de mortalidade e de complicações hospitalares, uma vez que um número desconhecido de desfechos observados fora do laboratório de hemodinâmica não é notificado no Registro CENIC, assim como ocorre em outros registros internacionais. ${ }^{19}$

\section{Limitações do estudo}

Este é um registro de ICP no cenário do IAMCSST, não contemplando o panorama completo do trata- 
mento do IAM, que também inclui pacientes tratados isoladamente com fibrinolíticos ou não submetidos a qualquer forma de reperfusão coronária (percutânea ou farmacológica). Outras limitações são o preenchimento e o envio voluntário de dados, com possíveis repercussões sobre a acurácia dos dados e a representatividade da casuística, que constituem limitações inerentes aos registros observacionais.

\section{CONCLUSÃO}

O Registro CENIC de 2006 a 2010 evidencia que a ICP no cenário do IAMCSST vem apresentando avanços ao longo do período analisado, embora de maneira heterogênea nas diferentes regiões do Brasil, mediante aumento das taxas de ICP primária e maior utilização de dispositivos de aspiração de trombos, os quais ainda não foram incorporados à rotina da ICP primária. Investimentos na formação e no treinamento de recursos humanos e implementação de protocolos de atendimento aos pacientes com IAMCSST constituem elementos essenciais para a otimização do tempo porta-balão e para a melhora dos resultados clínicos.

\section{CONFLITO DE INTERESSES}

Os autores declaram não haver conflito de interesses relacionado a este manuscrito.

\section{REFERÊNCIAS}

1. Brasil. Ministério da Saúde. DATASUS. Informações de saúde [Internet]. [citado 2011 mar. 14]. Disponível em: http://www2. datasus.gov.br/DATASUS/index.php?area $=02$

2. Zijlstra F, de Boer MJ, Hoorntje JC, Reiffers S, Reiber JH, Suryapranata $\mathrm{H}$. A comparison of immediate coronary angioplasty with intravenous streptokinase in acute myocardial infarction. N Engl J Med. 1993;328(10):680-4.

3. Vermeer $F$, Oude Ophuis AJ, van den Berg EJ, Brunninkhuis LG, Werter CJ, Boehmer AG, et al. Prospective randomised comparison between thrombolysis, rescue PTCA, and primary PTCA in patients with extensive myocardial infarction admitted to a hospital without PTCA facilities: a safety and feasibility study. Heart. 1999;82(4):426-31.

4. Widimský P, Groch L, Zelízko M, Aschermann M, Bednár F, Suryapranata $\mathrm{H}$, et al. Multicenter randomized trial comparing transport to primary angioplasty vs immediate thrombolysis vs combined strategy for patients with acute myocardial infarction presenting to a community hospital without a catheterization laboratory. The PRAGUE study. Eur Heart J. 2000;21(10): 823-31.

5. Widimský P, Budesinsky T, Vorac D, Groch L, Zelízko M, Aschermann M, et al.; 'PRAGUE' Study Group Investigators. Long distance transport for primary angioplasty vs. immediate thrombolysis in acute myocardial infarction. Final results of the randomized national multicentre trial - PRAGUE-2. Eur Heart J. 2003;24(1):94-104.

6. Andersen HR, Nielsen TT, Rasmussen K, Thuesen L, Kelbaek $H$, Thayssen $P$, et al. A comparison of coronary angioplasty with fibrinolytic therapy in acute myocardial infarction. $\mathrm{N}$ Engl J Med. 2003;349(8):733-42.

7. Mattos LA, Zago A, Chaves A, Pinto I, Tanajura L, Staico R, et al. Revascularização coronariana percutânea primária no infarto agudo do miocárdio: análise comparativa dos resultados imediatos em pacientes progressivamente longevos. Arq Bras Cardiol. 2001;76(1):53-62.

8. Keeley EC, Boura JA, Grines CL. Primary angioplasty versus intravenous thrombolytic therapy for acute myocardial infarction: a quantitative review of 23 randomized trials. Lancet. 2003;361(9351):13-20.

9. de Werf F, Bax J, Betriu A, Blomstrom-Lundqvist C, Crea F, Falk $\mathrm{V}$, et al. Management of acute myocardial infarction in patients presenting with persistent ST-segment elevation: the Task Force on the Management of ST-Segment Elevation Acute Myocardial Infarction of the European Society of Cardiology. Eur Heart J. 2008;29(23):2909-45.

10. Piegas LS, Feitosa G, Mattos LA, Nicolau JC, Rossi Neto JM, Timerman A, et al. Sociedade Brasileira de Cardiologia. Diretriz da Sociedade Brasileira de Cardiologia sobre Tratamento do Infarto Agudo do Miocárdio com Supradesnível do Segmento ST. Arq Bras Cardiol. 2009;93(6 Supl 2):e179-e264.

11. De Luca G, Stone GW, Suryapranata H, Laarman GJ, Menichelli $M$, Kaiser C, et al. Efficacy and safety of drug eluting stents in ST-segment elevation myocardial infarction: a meta-analysis of randomized trials. Int J Cardiol. 2009;133(2):213-22.

12. Mattos LA, Lemos Neto PA, Rassi A Jr, Marin-Neto JA, Sousa AGMR, Devito FS, et al. Diretrizes da Sociedade Brasileira de Cardiologia - Intervenção Coronária Percutânea e Métodos Adjuntos Diagnósticos em Cardiologia Intervencionista (II Edição - 2008). Arq Bras Cardiol. 2008;91(4 Supl 1):1-58.

13. Svilaas T, Vlaar PJ, van der Horst IC, Diercks GFH, de Smet BJGL, van den Heuvel AF, et al. Thrombus aspiration during primary percutaneous coronary intervention. $N$ Engl J Med. 2008;358(6):557-67.

14. Vlaar PJ, Svilaas T, van der Horst IC, Diercks GF, Fokkema ML, de Smet BJ, et al. Cardiac death and reinfarction after 1 year in the Thrombus Aspiration during percutaneous coronary intervention in Acute Myocardial Infarction Study (TAPAS): a 1-year follow-up study. Lancet. 2008;371(9638):1915-20.

15. Burzotta F, De Vita M, Gu YL, Isshiki T, Lefevre T, Kaltoft A, et al. Clinical impact of thrombectomy in acute ST-elevation myocardial infarction: an individual patient-data pooled analysis of 11 trials. Eur Heart J. 2009;30(18):2193-203.

16. De Luca G, Suryapranata H, Stone GW, Antoniucci D, Neumann FJ, Chiariello M. Adjunctive mechanical devices to prevent distal embolization in patients undergoing mechanical revascularization for acute myocardial infarction: a meta-analysis of randomized trials. Am Heart J. 2007;153(3):343-53.

17. Kushner FG, Hand M, Smith SC Jr, King SB 3rd, Anderson JL, Antman EM, et al. 2009 focused updates: ACC/AHA guidelines for the management of patients with ST-elevation myocardial infarction (updating the 2004 guideline and 2007 focused update) and ACC/AHA/SCAI guidelines on percutaneous coronary intervention (updating the 2005 guideline and 2007 focused update): a report of the American College of Cardiology Foundation/American Heart Association Task Force on Practice Guidelines. J Am Coll Cardiol. 2009;54(23): 2205-41.

18. European Association for Percutaneous Cardiovascular Interventions; Wijns W, Kolh P, Danchin N, Di Mario C, Falk $\mathrm{V}$, Folliguet $\mathrm{T}$, et al. The Task Force on Myocardial Revascularization of the European Society of Cardiology (ESC) and the European Association for Cardio-Thoracic Surgery (EACTS). Guidelines on myocardial revascularization. Eur Heart J. 2010; 31(20):2501-55.

19. Danchin N, Coste $P$, Ferrières J, Steg PG, Cottin $Y$, Blanchard D, et al. FAST-MI Investigators. Comparison of thrombolysis followed by broad use of percutaneous coronary intervention with primary percutaneous coronary intervention for STsegment-elevation acute myocardial infarction: data from the french registry on acute ST-elevation myocardial infarction (FAST-MI). Circulation. 2008;118(3):268-76. 